\title{
Effects of Blue Light on Mycelium Morphology, Citrinin Production and the Proportion of Sexual Spore of Monascus
}

\author{
Jing Wang ${ }^{\mathrm{a}}$, Changlu Wang ${ }^{\mathrm{b}}$, Mianhua Chen, Zhao Ban, Dong He, Hua Yang, Qian Zhang, \\ Yurong Wang, Fengjuan Li, Qimei Gu
}

Key Laboratory of Food Nutrition and Safety, Ministry of Education, College of Food Engineering and Biotechnology, Tianjin University of Science \& Technology, No. 29, the 13th Avenue, TEDA, Tianjin 300457, P. R. China

\begin{abstract}
To Monascus spp., light is not the required factor for its growth. But Monascus spp. has the capacity to sense and respond to light. This paper investigated the effects of blue light on growth and the changes of citrinin yield in Monascus 15. Our results demonstrated blue light was a stimulating signal for citrinin formation. Under the blue light illumination, the biomass of Monascus 15 was inhibited, but the citrinin yield increased when comparing with no light culture condition. Spores statistical results revealed that the blue light also influences the development of mycelium and spore formation.
\end{abstract}

Index Terms: blue light; citrinin; sexual spore; hyphal morphology; Monascus

(C) 2011 Published by MECS Publisher. Selection and/or peer review under responsibility of the Research Association of Modern Education and Computer Science.

\section{Introduction}

Although filamentous fungi are not able to use light for photosynthetic processes, illumination does play a role in morphology and circadian responses. For example, the study with Gigaspora gigantea showed that at low intensity light $\left(13.4 \mu \mathrm{E} \mathrm{s}^{-1} \mathrm{~m}^{-2}\right)$, maximum hyphal branching was achieved after $6 \mathrm{~h}$ light exposure, and at high intensity light $\left(10,800 \mu \mathrm{E} \mathrm{s}^{-1} \mathrm{~m}^{-2}\right)$, maximum hyphal branching was reached after only 8 min exposure [1]. The ascomycete Neurospora crassa has been proven to be a paradigm for photobiological, biochemical, and genetic studies of the enigmatic process of light regulation. In N. crassa, blue light regulates induction of carotenoid pigment production [2,3], protoperithecia (sexual fruiting body) formation [4], phototropism of perithecial beaks [5] and circadian rhythm. All of above mentioned phenomenon are abolished by mutations in wc-1 or wc-2 $[6,7,8]$. Trichoderma atroviride, a fungus used in biological control, sporulates in a synchronized manner following a brief pulse of blue light. A brief pulse of blue light $(400-480 \mathrm{~nm})$ given to a radially

* Corresponding author.

E-mail address: ${ }^{\mathrm{a} w a n g i n g .840917 @ 163 . c o m ;}{ }^{\mathrm{b}}$ changluwang2009@gmail.com 
growing colony in a Petri dish induces synchronous sporulation [9]. For T. atroviride, upon exposure to blue light, changes in membrane potential and in ATP levels, and a transient biphasic oscillation in intracellular cAMP levels, are observed [10].

Secondary metabolite productions usually commence late in the growth of the microbe, which is commonly associated with sporulation in microorganisms, including fungi [11].Secondary metabolites associated with sporulation can be divided into three broad categories: (I) metabolites that activate sporulation [12], (II) pigments required for sporulation structures [13], and (III) toxic metabolites secreted by growing colonies at the approximate time of sporulation $[14,15]$.

Monascus fermentation productions have been used as foods and medicines in the Orient for over 1000 years [16]. In taxonomy, the genus Monascus belongs to the family Monascaceae and to the order Eurotiales. Morphological, physiological, and biochemical characteristics, such as the shape of the colony, length of conidial chain, and production of pigment, have been considered suitable keys to the classification of Monascus. Based on physiological and morphological characteristics, there are six major species: M. pilosus, M. purpureus, M. ruber, M. floridanus, M. pallens, and M. sanguineus.

There are three kinds of well-known polyketides that are produced in Monascus post-fermentation stage, which are pigments, monacolin $\mathrm{K}$, citrinin, respectively. Monascus fermentation productions are now used as a natural colorant and a dietary supplement in China and Southeast Asia. As the citrinin has nephrotoxic and hepatotoxic properties in animal species tested, it sounded the alarm about the safety of monascus productions. Studies of the corrected factors on citrinin production by Monascus should be carried out. In this paper, we studied the effects of blue light on citrinin accumulation, sporulation formation and morphology changes of Monascus 15.

\section{Materials and methods}

\subsection{Microorganisms Culture Media and Conditions}

Monascus 15 was a high citrinin-producing strain, preserved by Tianjin University of Science and Technology, which was maintained on maltose agar medium for 4 to 6 days at $30^{\circ} \mathrm{C}$. The inoculum medium contained rice powder $30 \mathrm{~g}, \mathrm{KH}_{2} \mathrm{PO}_{4} 2.5 \mathrm{~g}, \mathrm{NaNO}_{3} 3 \mathrm{~g}$, and $\mathrm{MgSO}_{4} \cdot 7 \mathrm{H}_{2} \mathrm{O} 1 \mathrm{~g}$ in $1000 \mathrm{~mL}$ distilled water, and the initial $\mathrm{pH}$ of the medium was adjusted to 4.5 with lactic acid. The YES fermentation medium contained yeast extract $40 \mathrm{~g}$, sucrose $160 \mathrm{~g}$ per liter. The inoculum was incubated in a rotary shaker $(180 \mathrm{rpm})$ at $30^{\circ} \mathrm{C}$ for 2 days, and then a suspension of $10^{6}$ spores was transferred to a $60 \mathrm{~cm}$ Petri dish that contained $50 \mathrm{ml}$ YES medium, which was incubated under dark or blue light exposure $\left(0.009 \mathrm{~mW} / \mathrm{cm}^{2}\right)$ on a static illumination incubator for 9 days at $37^{\circ} \mathrm{C}$. The wavelength of blue-LED illumination is about $450 \mathrm{~nm}$.

\subsection{Determination of Citrinin}

Citrinin was determined by HPLC on a C18 column $(5 \mu \mathrm{m}, 250 \mathrm{~mm} \times 4.6 \mathrm{~mm})$ after filtration of fermentation with $0.22 \mu \mathrm{m}$ pore size filter. LC grade acetonitrile and methanol were purchased from Merck Company. Citrinin was obtained from Sigma Chemical Company. The mobile phase comprised of water and acetonitrile and methanol (70:10:20), the $\mathrm{pH}$ of which was adjust to 2.6 with $\mathrm{H}_{3} \mathrm{PO}_{4}$. The column temperature and flow rate was set at $30^{\circ} \mathrm{C}$ and $1.0 \mathrm{ml} / \mathrm{min}$, respectively. The detector used is fluorescence detector. The excitation and emission wavelength was set at 330 and $500 \mathrm{~nm}$, respectively. All data presented are the averages of results obtained from three independent measurements. 


\subsection{Biomass Measurement}

Monascus biomass was determined by gravimetric analysis after centrifugation of pigments extraction and drying of the precipitate in an oven to constant weight at $60^{\circ} \mathrm{C}$

\subsection{Observation of cell development}

YES liquid cultures inoculated with the same number of spores were incubated under dark and blue light for 7 or 9 days at $37^{\circ} \mathrm{C}$. After 3 days of culture, spores including asci and conidia were harvested with $0.9 \% \mathrm{NaCl}$ solution and counted using a counting chamber.

\section{Results}

\subsection{Effects of Blue Light on Production of Citrinin of Monascus 15}

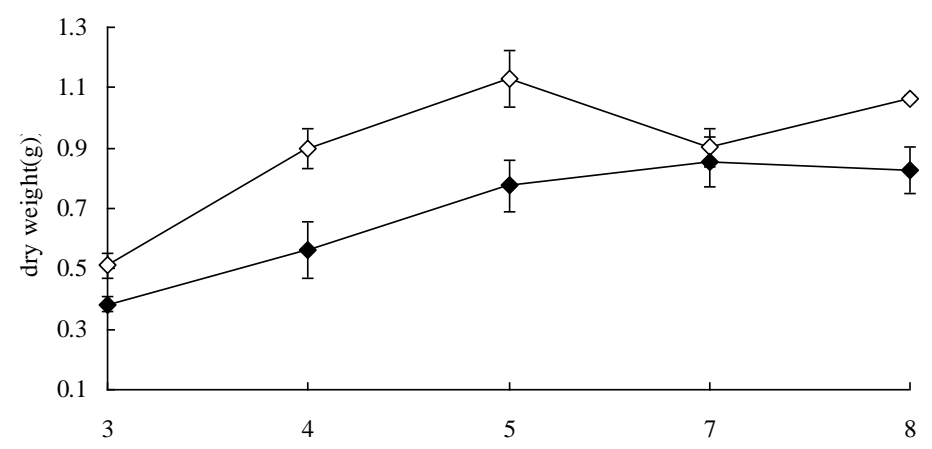

Fig. 1. Effect of light on cell dry weight. (Monascus $15 \mathrm{kept}$ in the dark $(\diamond)$; exposed to blue light $(\diamond)$.Values are the average of three independent experiments.)

The data showed in Fig.1 demonstrated that blue light cultivation decreased the cell dry weight of Monascus 15 when compared with the darkness fermentation. At the $5^{\text {th }}$ fermentation day, the highest dry weight was $1.130 \pm 0.094 \mathrm{~g}$. In the blue light group, the maximal biomass was $0.856 \pm 0.081 \mathrm{~g}$, which obtained at the $7^{\text {th }}$ fermentation day.

The Citrinin production was estimated in fermentation medium after 3, 4, 5 and 7 days of culture. From the data shown in Fig. 2, we knew that the peak value of citrinin under darkness fermentation was obtained after 3 days of cultivation, $648.4 \pm 17.6 \mu \mathrm{g} / \mathrm{ml}$. However, under the blue light irradiation, the maximum accumulation of citrinin appeared after 4 days of fermentation, which increased significantly (about 1.5 -fold) than that under no light cultivation (Fig. 2). The maximum value of the citrinin accumulation under the blue light fermentation was $987.9 \pm 98.9 \mu \mathrm{g} / \mathrm{ml}$. These results showed that blue light stimulated citrinin production in YES medium. On the other hand, at the telophase of fermentation, blue light accelerated the decomposition rate of citrinin, as well. 


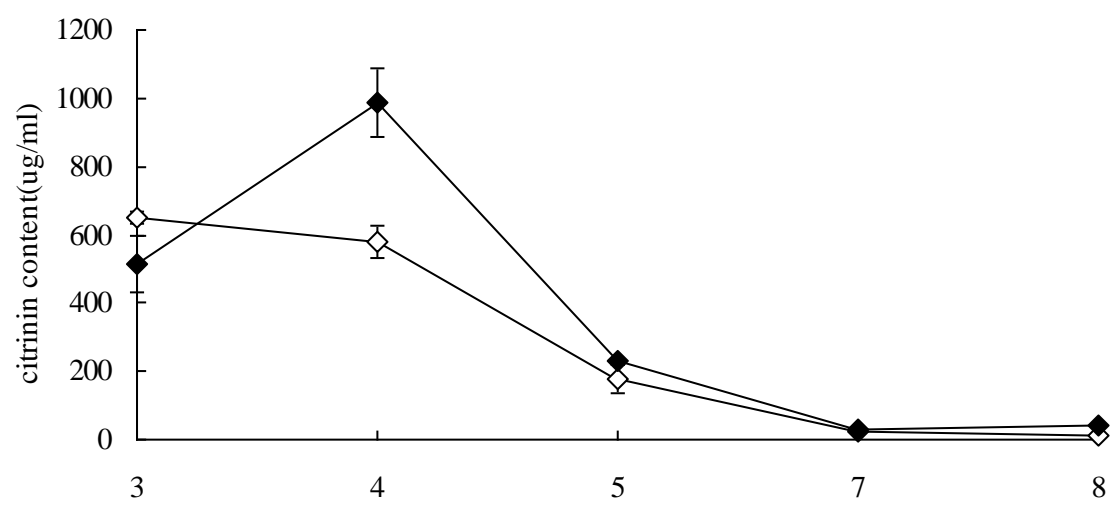

Fig. 2. Effect of blue light on citrinin content. (Monascus 15 cultivated under dark $(\diamond)$ ); exposed to blue light $(\diamond)$ ).Values are the average of three independent experiments. Error bars SD.)

\subsection{Effects of Blue light on Monascus mycelium morphology}

As shown in Fig. 3, Monascus 15 can sense and respond to blue light. When exposing to blue light, the aerial mycelium was short and rambling than that in the darkness culture condition. On the other hand, cultivation under the darkness, the mycelium was more exuberant, and stronger than that illuminated by blue light. At the central of the colonies, the colony color under the blue illumination was luteous (Fig. 3-C); whereas it appeared saffron yellow under the darkness (Fig. 3-D).

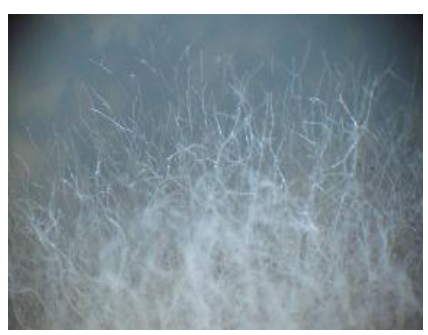

Fig. 3-A

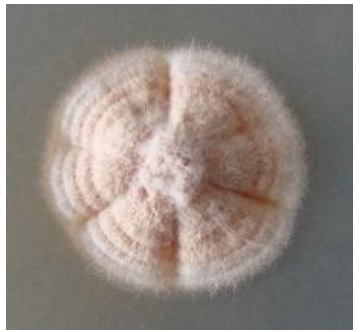

Fig. 3-C

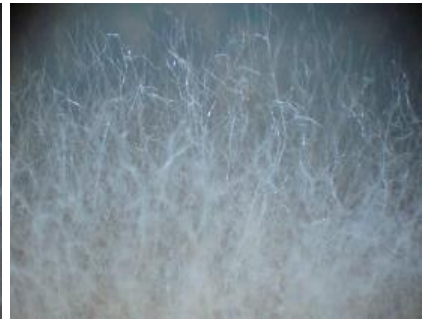

Fig. 3-B

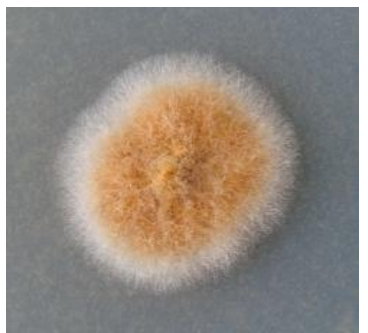

Fig. 3-D

Fig. 3. Morphological comparison of M15 under the blue light and dark culture conditions. (A, B, C, D: the aerial mycelium and the colony of M15 exposed to blue light and under dark culture conditions, respectively) 
The relationship of spores formation and blue light illumination was also investigated. At the 3rd day of culture, we got a small mycoderm by a puncher, the diameter of which was $1 \mathrm{~cm}$. Then, the spores in the small mycoderm, including asci and conidia, were harvested with $1 \mathrm{ml} 0.9 \% \mathrm{NaCl}$ solution and counted using a counting chamber (Table 1).

Table 1 Effect of light on spore formation in Monascus 15

\begin{tabular}{lllllll}
\hline \multirow{2}{*}{$\begin{array}{c}\text { Culture } \\
\text { days }\end{array}$} & conidia & asci & $\begin{array}{l}\text { Rate of asci } \\
\text { in spores }\end{array}$ & conidia & asci & $\begin{array}{l}\text { Rate of asci } \\
\text { in spores }\end{array}$ \\
\cline { 2 - 6 } & $1543 \pm 311$ & $588 \pm 38$ & 0.275 & $784 \pm 87$ & $318 \pm 39$ & 0.285 \\
$3^{\text {rd }}$ & $1298 \pm 34$ & $490 \pm 69$ & 0.273 & $1445 \pm 134$ & $735 \pm 69$ & 0.337 \\
$4^{\text {th }}$ & $1053 \pm 173$ & $245 \pm 29$ & 0.187 & $1372 \pm 177$ & $563 \pm 73$ & 0.288 \\
$5^{\text {th }}$ & $953 \pm 81$ & $147 \pm 21$ & 0.120 & $857 \pm 39$ & $318 \pm 34$ & 0.271 \\
$7^{\text {th }}$ & $833 \pm 72$ & $196 \pm 32$ & 0.177 & $759 \pm 43$ & $196 \pm 19$ & 0.206 \\
$8^{\text {th }}$ &
\end{tabular}

*Spore values are the average of three independent experiments \pm SD

Ascus formation was stimulated significantly by blue light exposure. The results showed that the number and rate of asci produced increased under blue light, whereas germination of conidia was inhibited by blue light. The maximum rate of asci in spores was $27.5 \%$ under dark, which appeared at the $3^{\text {rd }}$ day of fermentation. Whereas, cultivated with blue light, the maximum rate of asci was $33.7 \%$, which was at the $4^{\text {th }}$ day of fermentation. It was similar with the trendline of the citrinin content.

\section{Discussion}

In this study, we found that M15 can response to blue light, just like N. crassa did [8]. In our studies, blue light influences secondary metabolism and cell development, including mycelium and spore formation. Blue light stimulates the production of citrinin; meanwhile, it inhibits the biomass of monascus, which is consistent with the phenomenon that the aerial mycelium under the blue light illumination is more incompact and slimmer than that in the darkness. The maximum rate of asci in spores was consistent with the maximum citrinin content. Without the light illumination, the citrinin content reached maximum value $(648.4 \pm 17.6 \mu \mathrm{g} / \mathrm{ml})$ at the $3^{\text {rd }}$ day, whereas the maximum value appears at the $4^{\text {th }}$ day under the blue light cultivation, which was one day later than that in darkness. The maximum time inconsistency of citrinin accumulation with or without blue light illumination perhaps correlated with the circadian rhythms changes and other processes under the blue light illumination.

These observations indicate that blue light illumination influence the patterns of development of mycelium and spore formation, and then regulates the biosynthesis rate or the quantity of secondary metabolite in Monascus15. Why does Monascus secrete citrinin? Citrinin released to the external cultivation environment, is a kind of antimicrobial substance, which plays a role of self-protection, can inhibit the growth of other microorganisms. Our observation implied that another important function of the toxin maybe to act as a light protectant in order to create favorable conditions during the initial germination process. Illuminating with blue light, Monascus increased the biosynthesis of citrinin, a light protectant pigment in order to keep a steady-state for its own survival. In Neurospora crassa, the synthesis of carotenoids (photo-protective pigments) increased with white light irradiation. 


\section{Conclusion}

The members of the fungal kingdom can respond to the wavelengths of light from UV to far-red; however, until recently, only one photoreceptor class of the blue light sensors had been identified in N. crassa [17], Our data demonstrated that the blue light influenced the mycelium morphology, citrinin production and the proportion of sexual spore of Monascus 15, but the mechanisms behind blue light stimulation of citrinin and asci synthesis are still obscure.

\section{Acknowledgment}

This work was supported by the grant of the China National Natural Science Foundation (No. 20576100 \& 20776115).

\section{References}

[1] G. Nagahashi, D. Douds, Jr and M. Buee, "Light-induced hyphal branching of germinated AM fungal spores," Plant and Soil, vol. 219, pp. 71-79, 2000

[2] Harding, R.W., and Turner, R. V., "Photoregulation of the carotenoid biosynthetic pathway in albino and white collar mutants of Neurospora crassa," Plant Physiol, vol. 68, pp. 745-749, 1981

[3] Perkins, D. D., "Photoinduced carotenoid synthesis in perithecial wall tissue of Neurospora crassa," Fungal Genet. Newsl, vol. 35, pp. 38-39, 1988

[4] Degli-Innocenti, F., Pohl, U., and Russo, V. E. A, "Photoinduction of protoperithecia in Neurospora crassa by blue light," Photochem. Photobiol. Vol. 37, pp. 49-51, 1983

[5] Harding, R. W., and Melles, S., "Genetic analysis of the phototrophism of Neurospora crassa perithecial beaks using white collar and albino mutants," Plant Physiol, vol. 72, pp. 996-1000, 1983

[6] Perkins, D. D., Radford, A., Newmeyer, D., and Bjorkmann, M., "Chromosomal loci of Neurospora crassa," Microbiol. Rev, vol. 46, pp. 426-570, 1982

[7] Ballario, P., Vittorioso, P., Magrelli, A., Talora, C., Cabibbo, A., and Macino, "G. White collar-1, a central regulator of blue light responses in Neurospora, is a zinc finger protein,” EMBO J, vol.15, pp. 1650-1657, 1996

[8] H. Linden, P. Ballario, G. Macinol, "Blue Light Regulation in Neurospora crassa," Fungal Genetics and Biology, vol. 22, pp. 141-150, 1997

[9] Gressel, J. Galun, E., "Morphogenesis in Trichoderma: photoinduction and RNA," Dev Biol, vol. 15, pp. 575-598, 1967

[10] Gresik, M., Kolarova, N. \& Farkas, V., "Membrane potential, ATP, and cyclic AMP changes induced by light in Trichoderma viride,” Exp Mycol, vol. 12, pp. 295-301, 1988

[11] Sekiguchi, J., and G. M. Gaucher, "Conidiogenesis and secondary metabolism in Penicillium urticae," Appl. Environ. Microbiol, vol. 33, pp. 147-158, 1977

[12] Calvo, A. M., H. W. Gardner, and N. P. Keller, "Genetic connection between fatty acid metabolism and sporulation in Aspergillus nidulans,” J. Biol. Chem, vol. 276, pp. 20766-20774, 2001

[13] Kawamura, C., T. Tsujimoto, and T. Tsuge, "Targeted disruption of a melanin biosynthesis gene affects conidial development and UV tolerance in the Japanese pear pathotype of Alternaria alternata," Mol. PlantMicrobe Interact, vol. 12, pp. 59-63, 1999

[14] Hicks, J., J.-H. Yu, N. Keller, and T. H. Adams, "Aspergillus sporulation and mycotoxin production both require inactivation of the FadA Galpha protein-dependent signaling pathway," EMBO J, vol. 16, pp. 49164923, 1997 
[15] Trail F., Mahanti N, and Linz J., "Molecular biology of aflatoxin biosynthesis," Microbiology, vol. 141, pp. 755-765, 1995

[16] Wong, H. C, “Antibiotic and pigment production by Monascus purpureus," $\mathrm{Ph}$. D. Thesis, University of Georgia, 1982

[17] Sumathy Babitha, Julio C. Carvahlo, Carlos R. Soccol and A"shok Pandey, "Effect of light on growth, pigment production and culture morphology of Monascus purpureus in solid-state fermentation," World Journal of Microbiology and Biotechnology, vol. 24, No. 11, pp. 2671-2675, 2008 